Case Report

\title{
Kikuchi-Fujimoto Disease - A Clinical Enigma: Rare two Case Reports
}

\author{
Jayanti Khura',,$\underline{\text { Shalini Shakarwal }}{ }^{2}$, Vijay Kumar ${ }^{3}, \underline{\text { Sanjay Kumar }}{ }^{4}, \underline{\text { Pratap Singh }}^{5}$ \\ ${ }^{1}$ Senior Resident, ${ }^{5}$ Associate Professor, Department of Medicine, PGIMER and Dr. RML Hospital, New Delhi, India. \\ ${ }^{2}$ Ex-Assistant Professor, Department of Obs. \& Gynae. \\ ${ }^{3}$ Professor, Department of Pathology, ABVIMS \& Dr. RML Hospital, New Delhi, India. \\ ${ }^{4}$ Associate Professor, Department of Medicine, Lady Hardinge Medical College, New Delhi, India. \\ DOI: https://doi.org/10.24321/2349.7181.201914
}

\section{I $\quad \mathbf{N} \quad \mathbf{F} \quad \mathbf{O}$}

\author{
Corresponding Author: \\ Pratap Singh, Department of Medicine, PGIMER \\ and Dr. RML Hospital, New Delhi, India. \\ E-mail Id: \\ drpratapsingh@yahoo.co.in \\ Orcid Id: \\ https://orcid.org/0000-0001-6291-4287 \\ How to cite this article: \\ Khura J, Shakarwal S, Kumar V, Kumar S, Singh \\ P. Kikuchi-Fujimoto Disease - A Clinical Enigma: \\ Rare two Case Reports. J Adv Res Med 2019; \\ 6(3): 12-15. \\ Date of Submission: 2020-01-30 \\ Date of Acceptance: 2020-02-20
}

\section{$\begin{array}{llllllll}\mathbf{A} & \mathbf{B} & \mathbf{S} & \mathbf{T} & \mathbf{R} & \mathbf{A} & \mathbf{C} & \mathbf{T}\end{array}$}

Background: Kikuchi-Fujimoto Disease (KFD) is a rare disease and is commonly seen in Asian population. It usually presents with prolonged fever, leukopenia and persistent cervical lymphadenopathy. It is a benign disease with a female preponderance. The cause is unknown but many theories have been postulated, like autoimmune, inflammatory, infectious agents and molecular mimicry. Most of the patients will recover themselves without any sequelae, in six-month time. However, it may rarely also convert to autoimmune disease, like SLE. The recurrence rate is very low. It is important to consider it as a differential diagnosis in chronic persistent lymphadenopathy, like tuberculosis, lymphoma, HIV and autoimmune lymphadenitis.

Cases: Here we present a case of a 37-year-old female who initially went to a private practitioner with chief complaint of a persistent neck mass of approximately 6 months duration, multiple small joints pain and persistent fever. She underwent excisional biopsy for suspected lymphoma, but final pathology rendered a diagnosis of KFD. Second case also presented with multiple neck swelling and joint pains, suspect of SLE, connective tissue disorder or young-onset still's disease was made clinically but excisional biopsy revealed KFD. Both the patients improved with NSAIDS and low dose wysolone therapy.

Conclusion: The purpose of this article is not only to review the literature but also to create awareness of this entity in the differential diagnosis of persistent lymphadenopathy, especially for the general otolaryngologist in a community-based setting. In addition, this review would be beneficial for other practitioners as well, specifically paediatricians, infectious disease physicians, rheumatologists, pathologists, and medical oncologists.

Keywords: Kikuchi-Fujimoto Disease, KFD, Histiocytic Necrotizing Lymphadenitis, Lymphadenopathy, Systemic Lupus Erythematosus, SLE 


\section{Introduction}

Kikuchi Fujimoto disease is a rare self-limiting illness mainly affecting the cervical lymph nodes. It was first described in 1972 in Japan. ${ }^{1}$ It is also known as histiocytic necrotizing lymphadenitis or Kikuchi necrotizing lymphadenitis or phagocytic necrotizing lymphadenitis. It is mainly seen in Asia. Isolated cases have been reported in America and Europe. It is a disease of the young with a female preponderance. ${ }^{2}$ It is a disorder of unknown etiology, although infectious or autoimmune etiology has been postulated. ${ }^{3}$ Recurrence rate is about $3-4.5 \%$ and mortality is extremely rare. It frequently mimics other diseases such as malignant lymphoma, infectious etiologies, and autoimmune diseases, specifically Systemic Lupus Erythematosus (SLE), leading to unwanted treatment. Hence it is important not to miss the diagnosis of KFD. It has been incorrectly diagnosed as lymphoma in $30 \%$ cases. ${ }^{4}$ Herein we describe a female patient who presented with fever and generalized lymphadenopathy and was initially misdiagnosed as tuberculosis.

\section{Case: I}

A 37 years old female who was a resident of Ghaziabad, Uttar Pradesh, India, presented with complaints of fever for 6 to 8 months and joint pain of 2 months duration. The fever was insidious in onset, continuous, low grade without chills and relieved with oral medication. Since last 2 months she started having multiple joints pain involving bilateral small joints of hands, i.e. proximal and distal interphalangeal, metacarpophalangeal and bilateral elbow, wrist and knee joints. It was asymmetrical, without morning stiffness or any skin lesions. There was no history of urinary tract infection, acute gastro enteritis or any autoimmune disease. She also noticed painful swelling in neck, axilla and inguinal region, mainly in the posterior cervical area with slow enlargement. There was history of significant documented weight loss from $62 \mathrm{~kg}$ to $51 \mathrm{~kg}$, loss of appetite, easy fatiguability, generalized body ache. There was no history of cough, sputum, sore throat, hemoptysis, oral ulcer, skin lesions or photosensitivity. There was no history of drug addiction or high-risk behavior. She did not have past history of tuberculosis, jaundice or blood transfusion. Family history or personal history was not significant. She was previously taking treatment from a private clinic, wherein she was diagnosed with Tuberculosis and was advised Anti-Tubercular Treatment (ATT). She did not tolerate it and stopped it herself after taking it for 3 weeks.

On general physical examination she was thin built and was febrile to touch. She had bilateral cervical lymphadenopathy, mainly in the posterior triangle. The lymph nodes were 4 in number, each 1-2 cm in size, hard, tender, not fixed to the overlying skin. There were no skin color changes of the overlying skin either. She also had bilateral inguinal and axillary lymphadenopathy. Other than this she had no significant findings on general physical or systemic examination. Given the clinical picture of prolonged fever with multiple joint pain with generalized lymphadenopathy a list of differential diagnosis was made which included tuberculosis, lymphoma, connective tissue disease, HIV, sarcoidosis, still's disease and investigations were planned accordingly.

On investigation, the patient was anemic with $\mathrm{Hb}$ of $9.4 \mathrm{gm} /$ dl. She had a TLC of $3550 / \mathrm{mm}^{3}$ with $32 \%$ polymorphs, $58 \%$ lymphocytes, $6 \%$ monocytes and $4 \%$ eosinophils. She had a high ESR of $74 \mathrm{~mm}$ in $1^{\text {st }}$ hour. Peripheral smear demonstrated normocytic to microcytic RBCs with occasional target cells, platelets were adequate and a few reactive lymphocytes were seen. Mantoux test was negative with an induration of $5 \mathrm{~mm}$. Ultrasound of the abdomen demonstrated periportal lymphadenopathy and cholelithiasis. Fine needle aspiration of lymph node was done which revealed reactive lymphadenitis. HIV, HBsAg, anti-HCV serology was nonreactive. Serum ferritin was $720 \mathrm{ng} / \mathrm{mL}$ (normal value for females 12 to $150 \mathrm{ng} / \mathrm{mL}$ ) and serum ACE level was $120 / \mu \mathrm{L}$ (normal value for adults is 8 to 53 microliters). Autoimmune markers such as ANA, dsDNA, p \& c-ANCA, RA factor were all negative. Bone marrow aspiration and biopsy was normal. CECT of chest, abdomen and pelvis was done which demonstrated multiple lymph nodes in neck, axilla and mediastinum, porta and portocaval region and uterine fibroid in fundus (figure 1).

Cervical lymph node biopsy was done which revealed hyperplastic follicles and foci of necrosis containing scattered histiocytes, apoptotic cells, nuclear debris and fibrinous material located predominantly in sub-capsular location. A marked paucity of neutrophils was also noted. (figure 2 and 3 ). These features were consistent with reactive process with possibilities of necrotizing lymphadenitis (Kikuchi's disease) and autoimmune process \{mainly Systemic Lupus Erythematosus (SLE)\}. As autoimmune markers were negative and patient did not satisfy the Systemic Lupus International Collaborating Clinics (SLICC) classification criteria for SLE, it was excluded and a diagnosis of Kikuchi's Fujimoto disease was made. Patient was advised Non-Steroidal Anti-Inflammatory drugs (NSAID) and proton pump inhibitors following which she improved completely.

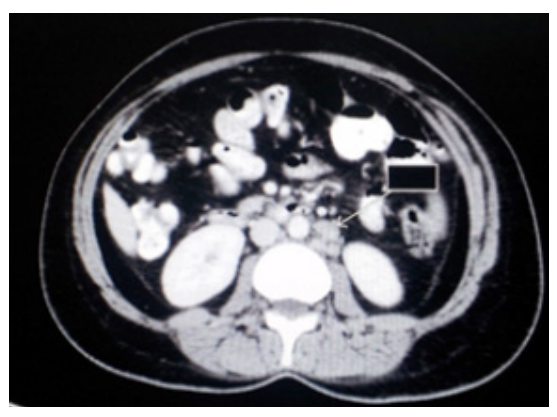

Figure I 


\section{Case Report: 2}

A 28-year-old young male patient presented with prolonged fever which was high grade, intermittent and associated with intermittent flushing of skin of face. He also had multiple painful lymphadenopathy and multiple large joints pain. He took ATT but there was no improvement in his clinical condition. All his investigations were within normal limits except lymph node biopsy which demonstrated Kikuchi disease. Patient was advised NSAID and wysolone after which his clinical condition improved.

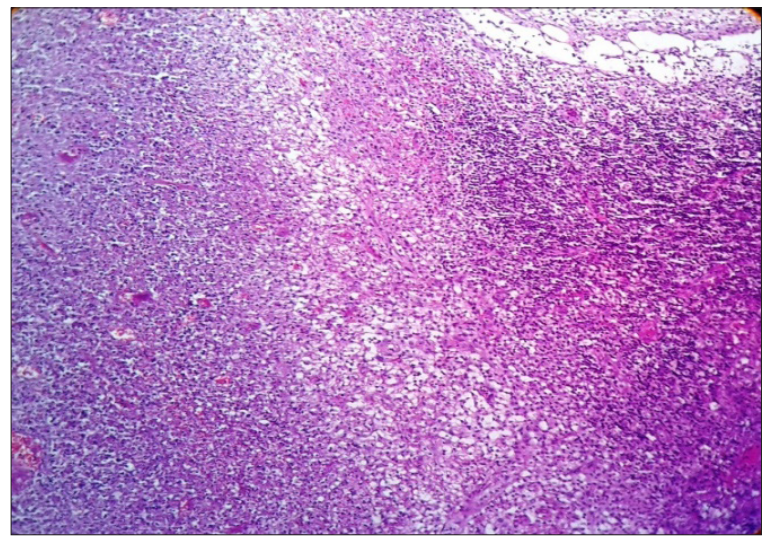

Histologically it is characterised by patchy areas of necrosis in the cortical and paracortical areas of the enlarged lymph node, together with nuclear debris or extensive karyorrhexis. ${ }^{7}$ Karyorrhectic foci consist of various types of histiocytes, plasmacytoid monocytes, immunoblasts and small and large lymphocytes. There is abundance of T cells with predominance of CD8+over CD4+ T cells. ${ }^{8}$

The pathophysiology of KFD is incompletely understood. Infectious and autoimmune causes have been proposed as etiology. ${ }^{2,9}$ Infectious causes include Mycobacterium

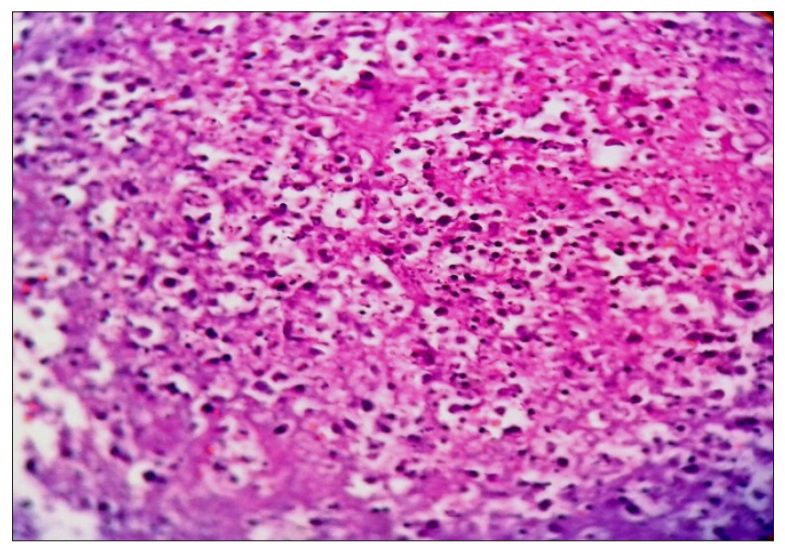

Figure 2\&3.Lymph node biopsy showing paracortical expansion with areas of necrosis (Hematoxylin \& Eosin), I0 x \& 100 x respectively

\section{Discussion}

KFD commonly presents with fever and lymphadenopathy as seen in the two cases above. It usually involves the cervical lymph nodes although other lymph node regions such as axillary, mediastinal, celiac and peripancreatic nodes may also be involved. ${ }^{5}$ The involved lymph nodes are painful and tender. The fever is prolonged ranging from 1 to 7 weeks with the temperature ranging from 38.6 to $40.5^{\circ} \mathrm{C} .{ }^{6}$ Other less common symptoms are headache, nausea, vomiting, malaise, fatigue, weight loss, arthralgias, myalgia, night sweats, rash, thoracic/ abdominal pain and symptoms associated with autoimmune disease. ${ }^{7}$ Skin manifestations of the disease include acneiform eruptions, facial erythema, papules, plaques, purpura, or nodules and when they are present, it is indicative of a more severe clinical presentation. ${ }^{2}$

The clinical picture of fever and lymphadenopathy includes many differential diagnoses such as lymphoma (Hodgkin's disease), SLE, TB, toxoplasmosis, metastatic carcinoma, cat-scratch disease, infectious mononucleosis and AIDS. Investigations must be done to exclude these diseases. Laboratory investigations will usually show nonspecific results characterised by leucopoenia (25-58 \%) and leucocytosis $(2-5 \%)^{8}$, anaemia, increased ESR, CRP, LDH and transaminitis. Imaging must be done to exclude other differential diagnosis. Definitive diagnosis is made by lymph node excision biopsy and histopathological examination. szulgai, Yesinia, Toxoplasma, Epstein-Barr virus, HHV 6, HHV 8, Parvovirus B 19, HIV and HTLV-1. There are a lot of studies showing association between SLE and KFD, but the relationship is not clearly understood. ${ }^{10,11,12}$ Histologically SLE differs from KFD in that it is associated with the presence of hematoxylin bodies, which are particles of denatured nuclear material. Londhey et al reported a case where a patient was initially diagnosed with KFD, but later went on to develop SLE, and suggested that it may precede, follow or coincide with the diagnosis of SLE. ${ }^{13}$ Hence patients diagnosed with KFD should be further investigated for autoimmune condition. Now there is also new proposed theory that KFD is a nonspecific hyperimmune reaction to a variety of infectious, chemical, physical and neoplastic agents but it needs further studies to prove.

Treatment is mainly symptomatic with NSAIDS which relieve the lymph node tenderness and fever. Glucocorticoid is used in severe extra nodal or generalized disease, multiple flares of bulky cervical lymphadenopathy and prolonged fever. Recurrence rate is about 3 to $4 \%$.

In conclusion, while dealing with a case of lymphadenopathy and fever, KFD should be in the list of differential diagnosis as it is a benign and self-limiting condition and can be treated simply with NSAIDs, requiring glucocorticoid only in severe cases. Excision biopsy must be performed in order to diagnose KFD and exclude other causes, such as malignant 
lymphoma (Hodgkin's disease), SLE, TB, toxoplasmosis, metastatic carcinoma, cat-scratch disease, infectious mononucleosis and AIDS. As KFD has a close relationship with SLE, further investigations must be done to rule out SLE and they should also be kept in regular follow up.

Financial Assistance: None

Conflict of Interest: None

\section{References}

1. Debley JS, Rozansky DJ, Miller ML, Katz BZ, Greene ME. Histiocytic necrotizing lymphadenitis with autoimmune phenomena and meningitis in a 14 years girl. Pediatrics 1996; 98(1): 30-33. Available from: https://pediatrics. aappublications.org/content/98/1/130 [PubMed/ Google Scholar].

2. Deaver D, Naghashpour M, Sokol L. Kikuchi-Fujimoto disease in the United States: three case reports and review of the literature. Mediterr J Hematol Infect Dis 2014; 6(1): e2014001. [PubMed/ Google Scholar].

3. Bosch X, Guilabert A. Kikuchi-Fujimoto disease. Orphanet J Rare Dis 2006; 1: 18. Available from: https://ojrd. biomedcentral.com/articles/10.1186/1750-1172-1-18 [Google Scholar].

4. Qadri F, Atkin GK, Thomas D, Das SK. Kikuchi's disease: an important cause of cervical lymphadenopathy. Clin Med 2007; 7: 82-84. [PubMed/ Google Scholar].

5. Santana A, Lessa B, Glarao L, Lima I, Santiago M. Kikuchi-Fujimoto's disease associated with systemic lupus erythematosus: case report and review of the literature. Clin Rheumato/ 2005; 24(1): 60-63. [PubMed/ Google Scholar].

6. Lee BC, Patel R. Kikuchi-Fujimoto disease: a 15-year analysis at a children's hospital in the United States. Clin Pediatr 2013; 52(1): 92-95. Available from: https:// utsouthwestern.pure.elsevier.com/en/publications/ kikuchi-fujimoto-disease-a-15-year-analysis-at-achildrens-hospit [Google Scholar].

7. Poulouse V, Chiam P, Poh W. Kikuchi's disease: a Singapore case series. Singapore Med J 2005; 46: 229232. [PubMed/ Google Scholar].

8. Bosch X, Guilabert A, Miquel R, Campo E. Enigmatic Kikuchi-Fujimoto disease: a comprehensive review. Am J Clin Pathol 2004; 122(1): 141-152. [PubMed/ Google Scholar].

9. Feder HM, Liu J, Rezuke WN. Kikuchi disease in Connecticut. J Pediatr 2014; 164(1): 196-200. [PubMed/ Google Scholar].

10. Kuculardali Y, Solmazgul E, Kunter E, Oncul O, Yildirim S, Kaplan M. Kikuchi-Fujimoto disease: analysis of 244 cases. Clin Rheumatol 2007; 26(1): 50-54. Available from: https://link.springer.com/article/10.1007/ s10067-006-0230-5 [PubMed/ Google Scholar].

11. Baenas DF, Diehl FA, Salinas MH, Riva C, Diller A,
Lemos P. Kikuchi-Fujimoto disease and systemic lupus erythematosus. Int Med Case Rep J 2016; 9: 163-167. [PubMed/ Google Scholar].

12. Goldblatt F, Andrews J, Russell A, Isenberg D. Association of Kikuchi-Fujimoto's disease with SLE. Rheumatology 2008; 47(4): 553-554. Available from: https://academic. oup.com/rheumatology/article/47/4/553/1790090 [PubMed/ Google Scholar].

13. Londhey VA, Buche AS, Kini SH, Rajadhyaksha GC. Kikuchi fujimoto disease and systemic lupus erythematosus-a rare association. The Journal of the Association of Physicians of India 2010; 58: 642-643. [PubMed/ Google Scholar]. 